\title{
Comparative Analysis of Rainfall with Estimated Evapotranspiration using Recurrent Neural Networks
}

\author{
H. Afzaal ${ }^{1}$, A.A. Farooque ${ }^{1}$, F. Abbas ${ }^{1}$, B. Acharya ${ }^{1}$, T. Esau ${ }^{2}$ \\ ${ }^{1}$ Faculty of Sustainable Design Engineering, University of Prince Edward Island, Charlottetown, Canada \\ ${ }^{2}$ Engineering Department, Dalhousie University, Agriculture Campus, Truro, Canada
}

\begin{abstract}
Evapotranspiration (ET) is key element in water balance as well as surface energy equation. Accurate estimation of ET provides useful information for water resource management, irrigation planning and crop sustainability. This study estimates the reference evapotranspiration (ET) with recurrent neural networks (RNN) namely long short term memory (LSTM) and Bidirectional LSTM. Four meteorological stations (North Cape, Summerside, Harrington and Saint Peters) were selected in Prince Edward Island. A new dataset namely PEI was computed by averaging climatic variables from all four sites to capture climatic variability from all parts of the province. The highest contributing climatic variables namely maximum air temperature and relative humidity were selected based on subset regression analysis as input variable for training (2011-2015) and testing (2016-2017) sets. The results suggested that the LSTM and Bidirectional LSTM are suitable methods to estimate reference ET with considerable accuracy as $\mathrm{R}^{2}>0.90$ for test set (2016-2017) for all site except Harrington. Testing period (2016-2017) root mean square errors were recorded in range of 0.38-0.58 mm/day for all sites. No major differences were observed in accuracy of LSTM and Bidirectional LSTM. Another objective of this study was to highlight the potential gap between reference ET and rainfall for agriculture sustainability in Prince Edward Island. The data from 2011-2017 highlights that the reference ET surpasses the rainfall affecting potential yield of major crops in the island. Viable options such as supplemental irrigation may needed to replenish the crop water requirements in drier months for agriculture sustainability.
\end{abstract}

